\title{
Improved Heart Rate Variability Signal Analysis from the Beat Occurrence Times According to the IPFM Model
}

\author{
Javier Mateo* and Pablo Laguna
}

\begin{abstract}
The heart rate variability (HRV) is an extended tool to analyze the mechanisms controlling the cardiovascular system. In this paper, the integral pulse frequency modulation model (IPFM) is assumed. It generates the beat occurrence times from a modulating signal. This signal is thought to represent the autonomic nervous system action, mostly studied in its frequency components. Different spectral estimation methods try to infer the modulating signal characteristics from the available beat timing on the electrocardiogram signal. These methods estimate the spectrum through the heart period (HP) or the heart rate (HR) signal. We introduce a new time domain HRV signal, the Heart Timing (HT) signal. We demonstrate that this HT signal, in contrast with the HR or HP, makes it possible to recover an unbiased estimation of the modulating signal spectra. In this estimation we avoid the spurious components and the low-pass filtering effect generated when analyzing HR or HP.
\end{abstract}

Index Terms - Heart rate variability, heart timing, IPFM model, nonuniform sampling, spectral analysis.

\section{INTRODUCTION}

$\mathbf{P}$ OWER spectral density (PSD) estimate of the heart rate variability (HRV) is commonly used as a noninvasive test of the neural control of the cardiovascular system, since it is related to the sympathetic and parasympathetic regulation of the sino-atrial node. The frequency domain analyzes have contributed to improve the understanding of the HRV since last two decades [1]-[4]. The HRV has been confirmed as a predictor of mortality following myocardial infarction [5], it has been studied in diabetics [6] and in cases of sudden cardiac death [7]. A review of the HRV research activity over the last two decades was presented in [8].

The integral pulse frequency modulation (IPFM) model has been assumed for many authors to explain the mechanisms used by the autonomic system to control the heart rate [9]-[15]. The IPFM model supposes a modulating signal, which when acting

Manuscript received September 7, 1998; revised March 9, 2000. This work was supported by the Comisión Interministerial de Ciencia y Technologia (CICYT), Spain, under Grant TIC97-0945-C02, by the Consejo Superior de Investigación y Desarrollo (CONSI+D) under Grant P40/98 from, and by the Diputación General de Aragón (DGA). Asterisk indicates corresponding author.

*J. Mateo is with the Grupo de Tecnologías de las Comunicaciones, Departamento de Ingeniería Electrónica y Comunicaciones, Centro Politécnico Superior, Universidad de Zaragoza, C/. María de Luna 3, 50015 Zaragoza, Spain (e-mail: jmateo@posta.unizar.es).

P. Laguna is with the Grupo de Tecnologías de las Comunicaciones, Departamento de Ingeniería Electrónica y Comunicaciones, Centro Politécnico Superior, Universidad de Zaragoza, 50015 Zaragoza, Spain.

Publisher Item Identifier S 0018-9294(00)06411-9. through the model generates the beat occurrence times. PSD methods try to infer the spectrum of the modulating signal from the beat occurrence times, usually from the Heart Rate (HR) or the Heart Period (HP) signals or by calculating the "Spectrum of Counts" (SPC) [13]. We will show how the HR, the HP, or the SPC's do not contain the same spectral information as the modulating signal according to the IPFM model. In this work, we introduce a new HRV time domain signal, the Heart Timing (HT) signal, used to deduce the characteristics of the heart control modulating signal. We will demonstrate that the proposed HT signal can be used to recover the spectrum of the modulating signal with no spurious contribution and no distortion, in contrast to the HR-, HP-, or SPC-related signals. We will extensively study these HRV-related signals as the first but fundamental stage in comparing the methods used in the estimation of the PSD of the HRV.

To show experimentally the validity of the estimation methods, and given that the spectrum of a real modulating signal of the heart activity is unknown, we have developed a controlled experiment with known modulating signals, $m(t)$. These signals come from reported autoregressive (AR) models representing real data [8], [16] and other computer generated signals. These $m(t)$ signals are used as inputs to the IPFM model to generate beat sequences. The beat sequences are analyzed with different HRV estimation methods and the obtained spectra are compared to the original ones of the $m(t)$ signals.

\section{The IPFM Model AND The Time Domain Signals}

The IPFM model is based on the hypothesis that the sympathetic and parasympathetic influences on the sino-atrial node can be represented by a single modulating signal, $m(t)$, and the beat trigger impulse is generated when the integral of this function reaches a threshold [10].

The beat occurrence time series can be generated by means of the IPFM model as

$$
k=\int_{0}^{t_{k}} \frac{1+m(t)}{T} d t
$$

where $k$ is an integer that represents the number of the $k$ th beat and $t_{k}$ is the occurrence time of the $k$ th beat [14]. We can see $(1+m(t)) / T$ as the instantaneous heart rate. $T$ is the mean of the RR intervals in the analyzing period and $m(t) / T$ represents the zero-mean dynamic part. This dynamic part is usually small as compared to the mean of heart rate $(m(t) \ll 1)$. It is considered that the first beat occurs at $t_{0}=0$ and that $m(t)$ is causal, 
hence, $m(t)=0$ if $t<0$. Another important consideration imposed on $m(t)$ is that it is a band-limited signal with negligible PSD over a frequency typically around $0.4 \mathrm{~Hz}$.

The objective of HRV PSD estimation methods is to infer the spectral properties of $m(t)$ from the heart beat positions, $t_{k}$. The choice of the time domain signal that best represents this information is the first problem in HRV analysis. So far, this problem has been addressed through the RR intervals, known as HP signals, through the inverse of the RR intervals, known as HR signals, or through the "event series," whose spectrum is known as Spectrum of Counts" (SPC) [11], [13]. Moreover, we will study the HT signal, recently proposed by us [17]. Also, we will distinguish between the unevenly spaced signals, measured at $t_{k}$ time instants (time referred), and the evenly spaced sequences, measured at each $k$ th beat (beat referred). The nonequispaced signals will be denoted in small caps and will be function of time. A final "s" will be appended to the notation of the equispaced signals that will be function of "beat number." Since these signals are not linearly related to the nonequispaced counterparts, its spectral properties are quite different. The following sections formalize these signal definitions and introduce the generalization of the continuous time IPFM model. This will allow the quantification of the inherent distortion of each HRV-related signal.

\section{A. Continuous Time IPFM Model Generalization}

The values of each time domain signal at the beat occurrence times, $t_{k}$, are all we know. However, we can find continuous time signals whose samples at $t_{k}$ coincide with these values. Of course, there are infinite continuous time signals that meet this requirement but we can generalize the IPFM model to define these signals with some logic. Thus, we can rewrite (1) as

$$
x=\int_{0}^{t(x)} \frac{1+m(t)}{T} d t
$$

$t(x)$ being a continuous function that solves the IPFM model equation, and whose samples at $x=k=1,2, \ldots$ are $t(k)=t_{k}$.

Now, we can define the different continuous signals and we can describe how these signals are related with the modulating signal, $m(t)$. We will obtain the unevenly sampled signals as functions of $t(x)$ or simply $t$, and the evenly spaced sequences will be functions of $x$.

\section{B. Time Domain Signals}

1) The HT signal: The formulation of the IPFM model given in (1) can be rewritten as

$$
h t\left(t_{k}\right)=k T-t_{k}=\int_{0}^{t_{k}} m(\tau) d \tau
$$

where $h t\left(t_{k}\right)$ defines the unevenly spaced samples of the HT signal and they can be easily calculated through the beat occurrence times [17], [18]. Each $h t\left(t_{k}\right)$ reflects the deviation of the position of each beat from the mean RR interval. We have emphasized unevenly spaced samples because behind this sampled signal we can find the continuous version of the HT signal. Using the generalization given by (2), the continuous $h t(t)$ signal can be written as

$$
h t(t)=x(t) \cdot T-t=\int_{0}^{t} m(\tau) d \tau .
$$

Thus, the continuous HT signal is defined simply as the integral of $m(t)$.

The $h t(t)$ signal is a straightforward HRV-related signal and, being linearly related with $m(t)$, it does not bring any distorted information with it. At this point, we should note that different observation times, leads to different $h t(t)$ functions since the mean heart rate and the dynamic heart rate also depend on the analyzing time. However, the instantaneous heart rate, $(1+m(t)) / T$, always can be obtained as

$$
\frac{1+m(t)}{T}=\frac{1+h t^{\prime}(t)}{T}
$$

for any observation time. $T$ always must be calculated as the mean of the RR intervals in the analyzing period.

From the spectral point of view, taking into account that $m(t)$ is causal and with zero-mean, its Fourier transform is

$$
H T(\omega)=\frac{M(\omega)}{j \omega}+\pi M(0) \delta(\omega)=\frac{M(\omega)}{j \omega}
$$

The Fourier transform of the $h t(t), H T(\omega)$, is the same as $M(\omega)$, except for the factor $1 / j \omega$ that comes from the integration. If $m(t)$ is band limited, $h t(t)$ is also band limited and if we knew $h t(t)$ or its regularly spaced samples satisfying the Nyquist criterion we could determine exactly $M(\omega)$ that is the focus of our interest. However, we only know the HT signal unevenly sampled at the beat occurrence times, $h t\left(t_{k}\right)$. All HRV-related signals are irregularly spaced and experience this problem. In Section III, an analysis of the irregular sampling problem will be shown.

The corresponding sequence of the HT signal is $h t s(k)=$ $k T-t_{k}$, and its continuous time generalization will be

$$
h t s(x)=x T-t(x) .
$$

It can be related with $h t(t)$ through (4) by means of the recurrence

$$
h t s(x)=h t((x))=h t(x T-h t s(x)) .
$$

We will use this kind of recursive relation to obtain an approximation of $h t s(x)$ from $h t(t)$, which will be all the more accurate, the more the iterative process is repeated. The initial value of $h t s(x)$ can be taken as $h t(x T)$. The recurrence expressed in (8) converges if $\left|h t^{\prime}(t)\right|<1$ [19]. This condition is always met in HRV because $\left|h t^{\prime}(t)\right|=|m(t)|<1$. If $m(t)$ is known then $h t(t)$ can be calculated by (4) and (8) permits us to study the spectral properties of $h t s(x)$ analytically. In the study of the different time-domain signals these relations are useful to show the inherent distortion of each HRV-related signal, even before any PSD estimation method has been applied.

2) The HP Signal: The classical time-domain heart period signal samples are defined as $h p\left(t_{k}\right)=t_{k}-t_{k-1}$ and following the continuous time generalization the heart period signal can 
be defined as $h p(t)=t(x)-t(x-1)$. The relation with the $h t(t)$ signal and, thus, with $m(t)$, can be established through (4) by the following recurrence relation:

$$
h p(t)=T-h t(t)+h t(t-h p(t)) .
$$

The corresponding sequence of the heart period signal is $h p s(k)=t_{k}-t_{k-1}$ and its spectrum is known as "interval spectrum" or "tachogram" [12]. Its continuous time generalization will be $h p s(x)=t(x)-t(x-1)$. It is related with the heart timing sequence, using (7), by the following relation:

$$
h p s(x)=T-h t s(x)+h t s(x-1) .
$$

3) The HR Signal: Another classically used time-domain signal is the heart rate signal whose samples are $h r\left(t_{k}\right)=1 /\left(t_{k}-t_{k-1}\right)$. The continuous heart rate signal can be defined as $h r(t)=1 /(t(x)-t(x-1))$. The relation with the $h t(t)$ signal can be established by the following recurrence relation:

$$
h r(t)=\frac{1}{T-h t(t)+h t\left(t-\frac{1}{h r(t)}\right)} .
$$

The corresponding sequence of the heart rate signal is $h r s(k)=1 /\left(t_{k}-t_{k-1}\right)$ and its spectrum is known as "Spectrum of the Inverse Intervals" [12]. Its continuous time generalization will be $h r s(x)=1 /(t(x)-t(x-1))$, and using (7), it is related with the heart timing sequence by

$$
h r s(x)=\frac{1}{T-h t s(x)+h t s(x-1)} .
$$

4) The Event Series: Once the beat occurrence times, $t_{k}$, are known, the most intuitive function which represents this kind of signal, is a impulse train of Dirac delta, each one placed at $t_{k}$. Then, the continuous function of time is

$$
\operatorname{spc}(t)=\sum_{k=-\infty}^{\infty} \delta\left(t-t_{k}\right)
$$

and its spectrum, known as "Spectrum of Counts" is

$$
\operatorname{SPC}(\omega)=\sum_{k=-\infty}^{\infty} e^{-j \omega t_{k}} .
$$

In the Appendix, it is shown that $\operatorname{SPC}(\omega)$ can be approximated at the frequency band of interest $(f<1 / 2 T)$ as

$$
\operatorname{SPC}(\omega) \approx \frac{1}{T}\{\delta(\omega)+M(\omega)+\operatorname{FM}(\omega)+M(\omega) * \operatorname{FM}(\omega)\}
$$

where $\operatorname{FM}(\omega)$ is given by

$$
\operatorname{FM}(\omega)=\mathcal{F} \mathcal{T}\left\{2 \cdot \cos \left(\frac{2 \pi}{T} t+\frac{2 \pi}{T} h t(t)\right)\right\} .
$$

Interpreting (15), we see that the spectrum of $\operatorname{spc}(t)$ is formed by a Dirac delta impulse at zero frequency plus the modulating signal spectrum, $M(\omega)$, plus two additional terms. The $\mathrm{FM}(\omega)$ term corresponds with a carrier of frequency $f_{s}=1 / T$, phase modulated by $h t(t)$ (or, equivalently, frequency modulated by $m(t)$ ). The convolution $M(\omega) * \mathrm{FM}(\omega)$ term is the spectrum of the same carrier modulated in amplitude and in frequency by $m(t)$. Once eliminated, the dc component, at the base band the spectrum is the one of modulating signal but with added spurious components resulting from the modulated carrier at $f_{s}=1 / T$.

The general analysis of this spectrum is highly complicated. The $\mathrm{FM}(\omega)$ and $M(\omega) * \mathrm{FM}(\omega)$ terms tend to compensate each other, obtaining spectral components lower than each one individually. The contaminating spectral contribution at the base band depends on the maximum amplitude of $m(t)$ and its spectral distribution. When $m(t)$ has a smooth spectrum, the contribution usually presents a hyperbolic shape, descending from the modulated carrier. The result is a small increment in the high frequency of the HRV spectrum estimation. However, if $m(t)$ has a sharp spectrum or it is a multitone signal the amplitude of spurious components may be comparable to those present in $m(t)$.

Fig. 1 shows graphically all these signals for $m(t)=$ $0.4 \cos \left(2 \pi \cdot f_{1} \cdot t\right)$ with $f_{1}=0.1 \mathrm{~Hz}$ and $T=1 \mathrm{~s}$. We have chosen an unusually large amplitude of $m(t)$ to show clearly the signal distortion. In this figure and throughout this paper the units of the time domain signals are scaled according to their relationship with the dimensionless $m(t)$ signal. Thus, HP-related signals are divided by $T$, HR signals are multiplied by $T$, and HT signals - since they are related with the integral of $m(t)$-are multiplied by $j \omega$ in the frequency domain, giving in all cases dimensionless magnitudes. This procedure allows obtaining homogeneous results from different time domain signals independentl of $T$. Also, the mean is removed from each time domain signal since the mean of $m(t)$ is zero. Regarding to the relative phase of each time domain signal respect to $m(t)$, HT signals will have a $90^{\circ}$ phase shift due to the integration, HR signals will have no phase shift, and HP signals will be $180^{\circ}$ out of phase due to the inversion. Thus, in this Fig. 1 shaded areas show the difference of $m(t)$ with each time domain signal. Circles represent the beat occurrence times.

\section{Two-Tone Harmonic Distortion}

We have made an algebraic study of the inherent distortion of the above signals when $m(t)$ is formed by two tones

$$
m(t)=a_{1} \cos \left(2 \pi f_{1} t+\varphi_{1}\right)+a_{2} \cos \left(2 \pi f_{2} t+\varphi_{2}\right) .
$$

In the case of the $\operatorname{spc}(t)$ signal, in [9] and [20]-[22], a quantification of the different spectral components is given when $m(t)$ is formed by a single-tone or multitone functions. Thus, for $m(t)$ given by (17), (13) can be given as

$$
\begin{aligned}
\operatorname{spc}(t)= & \frac{1}{T}+\frac{a_{1}}{T} \cos \left(\omega_{1} t+\varphi_{1}\right)+\frac{a_{2}}{T} \cos \left(\omega_{2} t+\varphi_{2}\right) \\
& +2 \sum_{n_{0}=1}^{\infty} \sum_{n_{1}=-\infty}^{\infty} \sum_{n_{2}=-\infty}^{\infty}\left(\frac{1}{T}+\frac{n_{1} f_{1}}{n_{0}}+\frac{n_{2} f_{2}}{n_{0}}\right) \\
& \cdot J_{n_{1}}\left(\frac{a_{1} n_{0}}{f_{1} T}\right) \cdot J_{n_{2}}\left(\frac{a_{2} n_{0}}{f_{2} T}\right) \\
& \cdot \cos \left(\frac{2 \pi n_{0}}{T} t+n_{1} \omega_{1} t+n_{2} \omega_{2} t+\phi\right)
\end{aligned}
$$



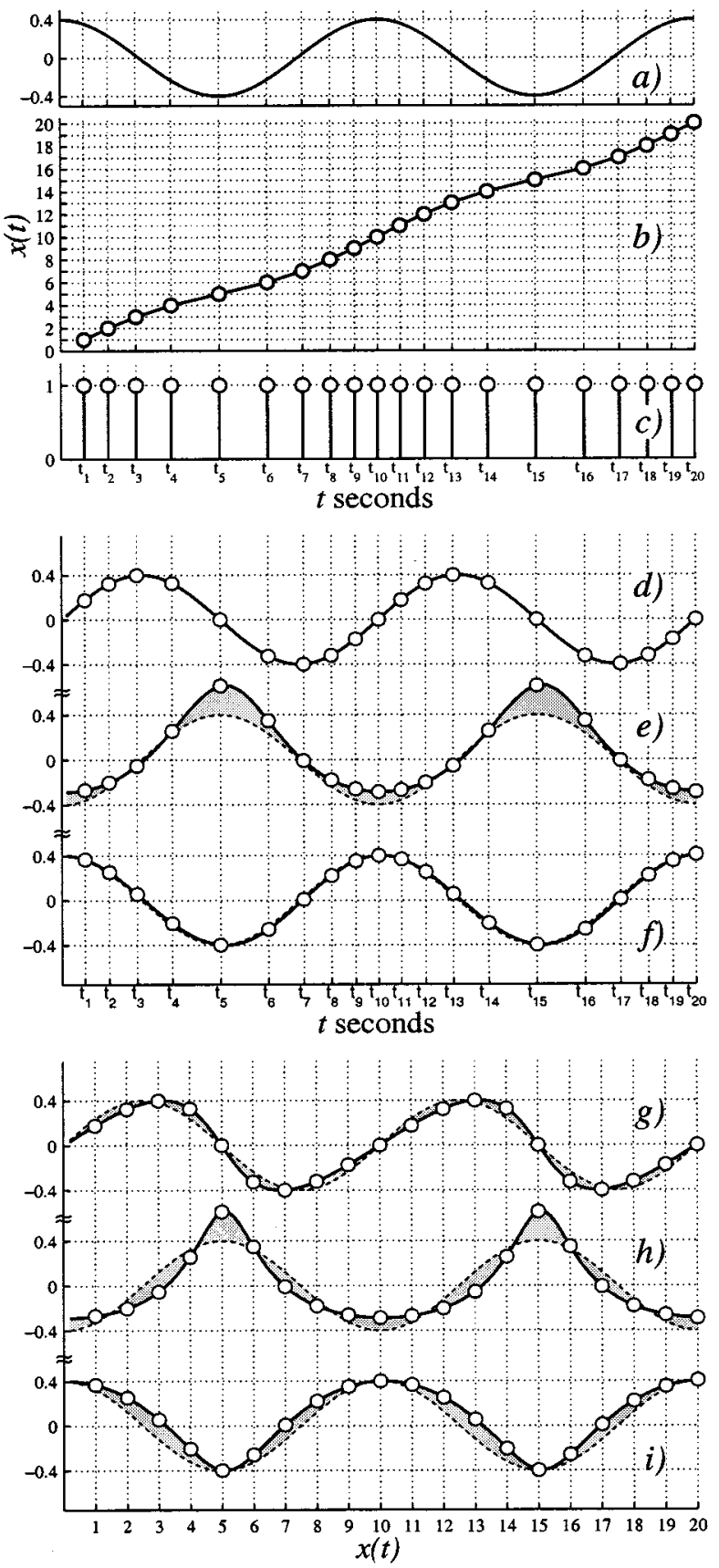

Fig. 1. Time domain signals for $m(t)=0.4 \cos \left(2 \pi f_{1} t\right)$ with $f_{1}=0.1 \mathrm{~Hz}$ and $T=1 \mathrm{~s}$. (a) Represents $m(t)$. (b) Represents $x(t)=\int_{0}^{t}(1+m(\tau)) / T \cdot d \tau$. (c) Represents $\operatorname{spc}(t)$. (d) Represents $2 \pi f_{1} \cdot h t(t)$. (e) Represents $h p(t) / T-1$. (f) Represents $h r(t) \cdot T-1$. (g) Represents $2 \pi f_{1} \cdot h t s(x)$. (h) Represents $h p s(x) / T-1$. (i) Represents $h r s(x) \cdot T-1$. Shaded areas show the differences between each time domain signal and $m(t)$ (the phase of $m(t)$ has been changed according to the phase shift of each represented signal). Circles represent the beat occurrence times.

with $T$ the mean heart period, $\omega_{x}=2 \pi f_{x}, J_{n}$ the first kind Bessel function of order $n$ and $\phi$ a complicated phase term. At the base band the most significative terms are given by $n_{0}=1$. In this case, Table I shows the amplitude of the main spectral components of the $\operatorname{spc}(t)$ signal multiplied by $T$ with $n_{1}$ and $n_{2}$ integers.

In the case of the $h t(t), h t s(x), h p(t), h p s(x), h r(t)$ and $h r s(x)$, the development is truly cumbersome but the basic
TABLE I

AmPlitude OF THE SPeCtral Components OF THE $T \cdot s p c(t)$ SignaL

\begin{tabular}{cc}
\hline Frequency & Spectral Amplitude of $T \cdot s p c(t)$ \\
\hline 0 & $\mathbf{1}$ \\
$f_{1} ; f_{2}$ & $a_{1} ; a_{2}$ \\
$\frac{1}{T}+n_{1} f_{1}+n_{2} f_{2}$ & $2 T\left(\frac{1}{T}+n_{1} f_{1}+n_{2} f_{2}\right) J_{n_{1}}\left(\frac{a_{1}}{f_{1} T}\right) J_{n_{2}}\left(\frac{a_{2}}{f_{2} T}\right)$ \\
\hline
\end{tabular}

idea is simple. We will use the continuous definitions given by (4) and (8)-(12). When the definition involves a recurrence relation, it is developed several times. The number of times depends on the approximation level that we want to reach but the complexity increases considerably with each iteration. The next stage is to develop its Taylor series expansion with respect to the $a_{1}$ and $a_{2}$ variables, supposing $a_{1}, a_{2} \ll 1$. Finally, the series is reorganized in terms of each spectral component.

Table II shows the results obtained. In the case of the HP signals the amplitudes have been divided by $T$, and in the case of the HR signals, they have been multiplied by $T$. Also, the variables $\nu_{1}=\pi f_{1} T$ and $\nu_{2}=\pi f_{2} T$ have been introduced for the sake of simplicity. Except for the $h t(t)$, all the other signals are nonlinearly related with $m(t)$. They have spectral components at $m f_{1}+n f_{2}, m, n$ being any pair of integers. We show only the first significant term of the amplitude of the main spectral components. In the case of $h t(t)$ signal, Table II shows the exact amplitude of the spectral components, which correspond exactly to the ones present in $m(t)$.

Fig. 2 shows the amplitude of the spectral components of the studied signals for $m(t)=0.1 \cos (2 \pi \cdot 0.1 \cdot t)+0.1 \cos (2 \pi$. $0.25 \cdot t)$ and $T=1 \mathrm{~s}$. This figure, Table I, and Table II permit us to extract some preliminary conclusions about the inherent properties of the time domain signals.

- The spectrum of $m(t)$ can be exactly recovered by $j \omega$. $H T(\omega)$

- All HP or HR signals attenuate the original tone amplitude, all the more when its frequency increases. Thus, they have intrinsically a low-pass filtering effect.

- The harmonics are lower in HR signals than in HP and they are lower in $h p(t), h r(t)$, and $h t(t)$ than in $h p s(x), h r s(x)$, and $h t s(x)$. See, also, Fig. 1.

- Except for the $h t(t)$ signal, $h r(t)$ presents the smallest spurious contribution. However, $h r s(x)$ makes a large spurious contribution at the intermodulation frequencies, specially at $f_{2}-f_{1}$.

- The spurious contribution of $\operatorname{spc}(t)$ has a different origin and is noticeable at frequencies greater than $1 / 4 T$.

\section{THE IRREGULAR SAMPLING}

Whichever signal related with HRV is inherently irregularly sampled. In the analysis of the HRV, three main alternatives have been used to get around this problem: By assuming that HRV signals are evenly sampled, by using direct spectral estimation methods from the irregular sampled signal and by using interpolating methods to recover an evenly sampled signal from the irregularly spaced samples prior to the PSD estimation. 
TABLE II

MAIN SPECTRAL COMPONENTS OF THE $h t^{\prime}(t), h t s^{\prime}(x), h p(t) / T, h p s(x) / T, T \cdot h r(t)$, AND $T \cdot h r s(x)$ SignalS

\begin{tabular}{|c|c|}
\hline Frequency & Exact spectral amplitude of $j \omega \cdot \mathcal{F} \mathcal{T}\{h t(t)\}$ \\
\hline$f=0 ; f_{1} ; f_{2}$ & $0 ; a_{1} ; a_{2}$ \\
\hline$f=2 f_{1} ; 2 f_{2}$ & $0 ; 0$ \\
\hline$f=f_{1} \pm f_{2}$ & \\
\hline Frequency & First significant term of the spectral amplitude of $j \omega \cdot \mathcal{F T}\{h t s(x)\}$ \\
\hline $\begin{array}{l}f=0 ; f_{1} ; f_{2} \\
f=2 f_{1} ; 2 f_{2}\end{array}$ & $\begin{array}{c}0 ; a_{1} ; a_{2} \\
a_{1}^{2} ; a_{2}^{2}\end{array}$ \\
\hline$f=f_{1} \pm f_{2}$ & $\frac{a_{1} a_{2}}{2} \frac{\left(f_{1} \pm f_{2}\right)^{2}}{f_{1} f_{2}}$ \\
\hline Frequency & First significant term of the spectral amplitude of $\mathcal{F} \mathcal{T}\{h p(t)\} / T$ \\
\hline$f=0 ; f_{1} ; f_{2}$ & $1 ; \frac{a_{1}}{\nu_{1}} \sin \nu_{1} ; \frac{a_{2}}{\nu_{2}} \sin \nu_{2}$ \\
\hline$f=2 f_{1} ; 2 f_{2}$ & $\frac{a_{1}^{2}}{2 \nu_{1}} \sin \nu_{1} ; \frac{a_{2}^{2}}{2 \nu_{2}} \sin \nu_{2}$ \\
\hline$f=f_{1} \pm f_{2}$ & $\frac{a_{1} a_{2}}{2 \nu_{1} \nu_{2}} \sqrt{\left(\nu_{1} \pm \nu_{2}\right)\left(\nu_{1} \sin ^{2} \nu_{2} \pm \nu_{2} \sin ^{2} \nu_{1}\right) \mp \nu_{1} \nu_{2} \sin ^{2}\left(\nu_{1} \mp \nu_{2}\right)}$ \\
\hline Frequency & First significant term of the spectral amplitude of $\mathcal{F T}\{h p s(x)\} / T$ \\
\hline$f=0 ; f_{1} ; f_{2}$ & $1 ; \frac{a_{1}}{\nu_{1}} \sin \nu_{1} ; \frac{a_{2}}{\nu_{2}} \sin \nu_{2}$ \\
\hline$f=2 f_{1} ; 2 f_{2}$ & $\frac{a_{1}^{2}}{2 \nu_{1}} \sin 2 \nu_{1} ; \frac{a_{2}^{2}}{2 \nu_{2}} \sin 2 \nu_{2}$ \\
\hline$f=f_{1} \pm f_{2}$ & $\frac{a_{1} a_{2}\left(\nu_{1} \pm \nu_{2}\right)}{2 \nu_{1} \nu_{2}} \sin \left(\nu_{1} \pm \nu_{2}\right)$ \\
\hline Frequency & First significant term of the spectral amplitude of $T \cdot \mathcal{F T}\{h r(t)\}$ \\
\hline$f=0 ; f_{1} ; f_{2}$ & $1 ; \frac{a_{1}}{\nu_{1}} \sin \nu_{1} ; \frac{a_{2}}{\nu_{2}} \sin \nu_{2}$ \\
\hline$f=2 f_{1} ; 2 f_{2}$ & $\frac{a_{1}^{2}}{2 \nu_{1}^{2}} \sin \nu_{1} \sqrt{\sin ^{2} \nu_{1}-\nu_{1} \sin 2 \nu_{1}+\nu_{1}^{2}} ; \frac{a_{2}^{2}}{2 \nu_{2}^{2}} \sin \nu_{2} \sqrt{\sin ^{2} \nu_{2}-\nu_{2} \sin 2 \nu_{2}+\nu_{2}^{2}}$ \\
\hline$f=f_{1} \pm f_{2}$ & $\frac{a_{1} a_{2}}{2 \nu_{1} \nu_{2}} \sqrt{4 \sin ^{2} \nu_{1} \sin ^{2} \nu_{2}+\nu_{1}\left(\nu_{1} \pm \nu_{2}-2 \sin 2 \nu_{1}\right) \sin ^{2} \nu_{2} \pm \nu_{2}\left(\nu_{1} \pm \nu_{2} \mp 2 \sin 2 \nu_{2}\right) \sin ^{2} \nu_{1} \mp \nu_{1} \nu_{2} \sin ^{2}\left(\nu_{1} \mp \nu_{2}\right)}$ \\
\hline Frequency & First significant term of the spectral amplitude of $T \cdot \mathcal{F T}\{h r s(x)\}$ \\
\hline$f=0 ; f_{1} ; f_{2}$ & $1 ; \frac{a_{1}}{\nu_{1}} \sin \nu_{1} ; \frac{a_{2}}{\nu_{2}} \sin \nu_{2}$ \\
\hline$f=2 f_{1} ; 2 f_{2}$ & $\frac{a_{1}^{2}}{2 \nu_{1}^{2}}\left(\sin ^{2} \nu_{1}-\nu_{1} \sin 2 \nu_{1}\right) ; \frac{a_{2}^{2}}{2 \nu_{2}^{2}}\left(\sin ^{2} \nu_{2}-\nu_{2} \sin 2 \nu_{2}\right)$ \\
\hline$f=f_{1} \pm f_{2}$ & $\frac{a_{1} a_{2}}{2 \nu_{1} \nu_{2}}\left(\cos \left(\nu_{1} \pm \nu_{2}\right)-\cos \left(\nu_{1} \mp \nu_{2}\right)+\left(\nu_{1} \pm \nu_{2}\right) \sin \left(\nu_{1} \pm \nu_{2}\right)\right)$ \\
\hline
\end{tabular}

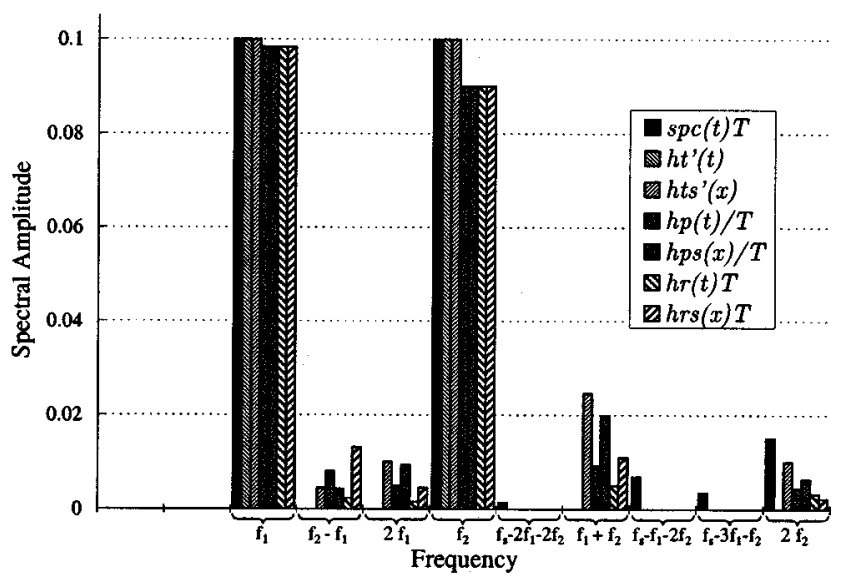

Fig. 2. Amplitude of the main spectral components of the time-domain signals for $m(t)=0.1 \cos \left(2 \pi f_{1} \cdot t\right)+0.1 \cos \left(2 \pi f_{2} \cdot t\right)$ and $T=1 \mathrm{~s} .\left(f_{1}=0.1 \mathrm{~Hz}\right.$. $f_{2}=0.25 \mathrm{~Hz}$. and $f_{s}=1 / T=1 \mathrm{~Hz}$.)

\section{A. HRV Signals Assumed to be Evenly Sampled}

This is the simplest and quickest method, but the assumption of regularly spaced samples when these are not regularly spaced, causes distortion and generates spurious harmonics. The obtained spectra will be that corresponding to the $h p s(x), h r s(x)$, and $h t s(x)$ signals.

\section{B. Direct Spectral Estimation Methods}

When a signal is sampled, the spectrum of the sampled signal is the result of the convolution of the original spectrum with $\sum_{k=-\infty}^{\infty} e^{-j \omega t_{k}}$ which is a well-known result. When $t_{k}$ is regularly spaced, this summation is a Dirac delta train spaced every $2 \pi / T$ due to orthogonality of exponential functions. In this case, if the signal satisfies the Nyquist criterion, there is no aliasing and the spectrum of sampled signal is the periodic repetition of the spectrum of the original signal. When the samples are not regularly spaced, the orthogonality of exponentials is lost and this summation is not a Dirac delta train. This summation is the above described SPC. Thus, given a continuous time signal $h x(t)$ ( $h x$ can be $h t, h p$, or $h r$ ) whose spectrum is $H X(\omega)$, the corresponding direct estimated spectrum is

$$
H X_{s}(\omega)=H X(\omega) * \sum_{k=-\infty}^{\infty} e^{-j \omega t_{k}}=H X(\omega) * \operatorname{SPC}(\omega)
$$

Leaving out the terms of minor significance, $\operatorname{FM}(\omega)$ and $M(\omega) * \operatorname{FM}(\omega)$, in (15) the direct estimated spectrum may be approximated as

$$
H X_{s}(\omega) \approx H X(\omega)+H X(\omega) * M(\omega)
$$

In $\mathrm{HRV}$, the second term may be significative and $H X_{s}(\omega)$ may be rather different from the original one $H X(\omega)$. We should note that in HRV the position of the samples is not independent of the signal and, thus, the same information is twice present: in the time-domain signal, whose spectrum is $H X(\omega)$, and in the position of the samples, whose spectrum is $\operatorname{SPC}(\omega)$. 


\section{Interpolation Methods}

Spline interpolation obtains a continuous piecewise polynomial reconstruction of its irregularly spaced samples [23]. From the evenly spaced samples of the signal, a usual PSD estimation can be carried out. Moreover, in the case of the HT signal, the obtained spline can be algebraically differentiated and in this way, we can obtain the instantaneous heart rate from (5).

The interpolation can be seen as a time-variant filter that acts with different frequency response as a function of the space between interpolated samples. Thus, the spectrum obtained by means of this method is filtered with a time-variant filter $F(\omega, t)$. It is true that the filter response depends on the time distribution of the samples. However, an estimation may be made for the filter response. The impulsive response may be calculated by interpolating a unitary pulse at $t=0$, preceded and followed by zeros regularly spaced at $T_{0}$. Intermediate points are obtained by interpolating along with the interpolation factor. The filter response is calculated as the spectrum of the impulsive response. This response will affect at those interbeat areas of heart period $T_{0}$. Since heart period changes from beat to beat, the filtering effect changes and becomes time varying.

Fig. 3 shows the estimated impulsive response and the spectrum for linear, fourth-order "cubic" spline and fourteenth-order spline interpolation methods.

The linear interpolation has a cutoff frequency that goes from $0.36 / T_{0} \mathrm{~Hz}$ when the interpolation factor is two and goes to $0.32 / T_{0} \mathrm{~Hz}$ when the interpolation factor is 16 or higher. The cubic spline method has a cutoff frequency of $0.44 / T_{0} \mathrm{~Hz}$ and it has a negligible dependence on the interpolation factor. Finally, the cutoff frequency obtained for the fourteenth-order spline is $0.48 / T_{0} \mathrm{~Hz}$. The linear method has the lowest cutoff frequency and the highest sidelobes as expected due to its inferior performance. Increasing the order of the spline, we establish a closer response to an ideal filter. However, experimentally we do not get any improvement with orders greater than fourteen, due to the finite numerical precision and round off errors. Because the cutoff frequency is relative to $1 / T_{0}$ frequency, in absolute terms of frequency, the filtering effect will be more significant when the heart rate is low.

\section{PSD ESTIMATION METHODS OF HRV}

In the study of HRV, different spectral analysis methods are used [11]-[16]. We will show an experimental comparative analysis of the time-domain alternatives presented in this paper in combination with the main different PSD estimation methods used in HRV. We have studied the following methods.

\section{A. "Spectrum of Counts" (SPC)}

This method is computed using (14) and it has been used by many authors. Its importance is practical as an estimation method of long-standing and theoretical for its relation with the irregular sampling problem. We will show how its performance is effective with HRV signals but this method introduces contamination at high frequencies. Thus, if the modulating signal has enough power at frequencies greater than $1 / 4 T$, the results present a high-frequency spurious contribution.
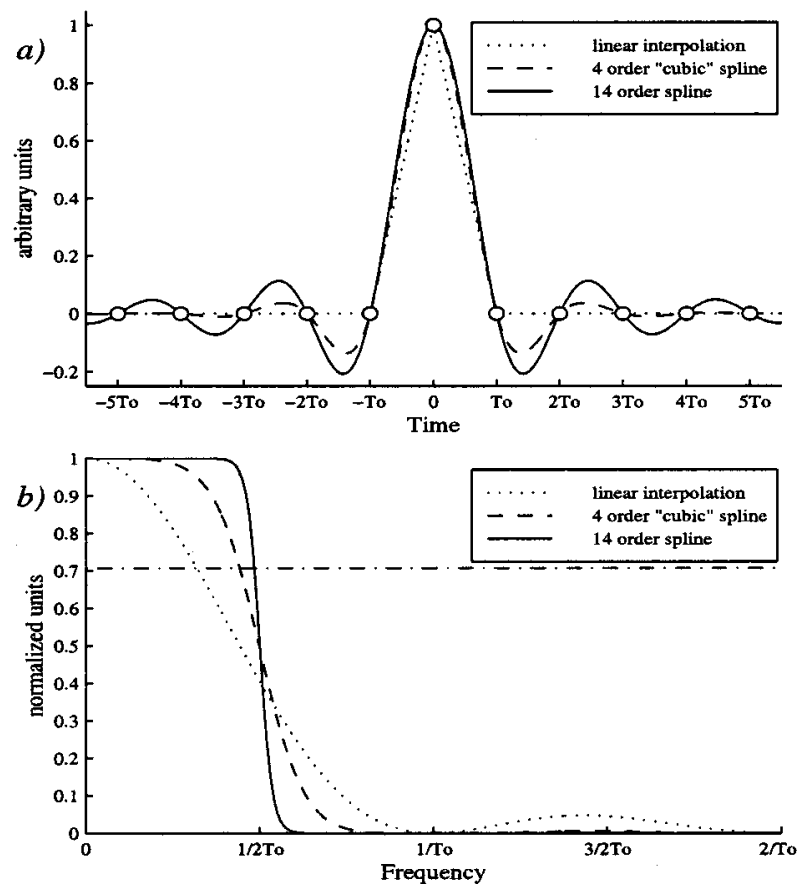

Fig. 3. Linear interpolation with interpolation order 16, cubic spline and fourteenth-order spline interpolation methods. (a) Impulsive response. (b) Frequency response.

\section{B. Low-Pass Filtered Event Series (LPFES)}

The LPFES, lpfes $(t)$, is the signal obtained by low-pass filtering the $\operatorname{spc}(t)$ signal [10], [11]. This signal is calculated at regular intervals of $T \mathrm{~s}$. and then, the fast Fourier transform (FFT) is taken to compute the spectrum. From this point of view, the obtained spectrum would be $\operatorname{LPFES}(\omega)=H_{F}(\omega) \cdot \operatorname{SPC}(\omega)$, where $H_{F}(\omega)$ is the response of the filter. Also, the authors [11] introduced an improvement inserting a "dummy pulse" at the midpoint of each RR interval. In this way, the carrier frequency involved in SPC is virtually doubled and the nonlinear contribution resulting from the carrier modulation is minimized. However, this pulse introduces an effect like linear interpolation giving a low-pass filtered spectrum as we will show. This method was originally implemented [11] dealing with real time and hardware limitations. Originally the filter had a cosine squared spectral response and was computed with relative low time resolution. In this paper, this method has been implemented with an ideal filter response with a cut off frequency of $0.5 \mathrm{~Hz}$, and with the insertion of a "dummy pulse" at the midpoint of each RR interval. Note that without the dummy pulse the obtained results would be essentially the same that with the SPC method.

\section{DFT of the Sequences (FHP, FHR, FHT)}

The power spectrum is directly computed by taking the FFT of the different sequences $h p s(k), h r s(k)$ or $h t s(k)$. The irregular sampling is overcome assuming that HRV signals are evenly sampled. This is the simplest and quickest method, but the assumption of regular spaced samples when these are not regularly spaced, causes distortion and generates spurious harmonics. When the $h p s(k)$ sequence is used, this method is known as "Spectrum of Intervals" or "Tachogram" [13], [14]. With the $h r s(k)$ sequence, this method is known as "Spectrum of Inverse Intervals" [13], 
[14]. We will also present this method applied to the $h t s(k)$ sequence to show the harmonic distortion caused by supposing regular sampling when there was irregular.

\section{Lomb Method (LHP, LHR, LHT)}

The Lomb-Scargle periodogram is a direct method to calculate the power spectrum of a unevenly sampled signal [20], [24], [25]. A fast algorithm is proposed in [26], [27] to calculate the Lomb periodogram. In HRV signals, the results obtained by the generalized periodogram defined as $\operatorname{PHX}(\omega)=$ $(1 / N) \sum_{k=1}^{N} h x\left(t_{k}\right) e^{-j \omega t_{k}}$ and by the Lomb method are similar [24]. The $\operatorname{PHX}(\omega)$ spectrum is exactly that obtained by direct estimation in (19). In this paper, the Lomb-Scargle periodogram has been used as the representative of the direct spectral estimation methods.

\section{E. Berger Method (BHP, BHR, BHT)}

In [14], an algorithm was presented so as to minimize the spurious spectral components. This method generates a stepwise heart rate signal from the instantaneous heart rate. This signal is sampled at $4 \mathrm{~Hz}$ and then is convolved with a rectangular window of $0.5 \mathrm{~s}$ of duration. This method can be seen as a zero-order hold system, whose holding times are irregularly spaced at the beat occurrence times and recovers a regular spaced signal by filtering and sampling the stepwise signal. The obtained spectrum is then corrected by multiplying it by the inverse of the filtering response caused by the convolution with the rectangular window. We extended this algorithm, originally for the $h r\left(t_{k}\right)$ signal, to the $h p\left(t_{k}\right)$ and $h t\left(t_{k}\right)$ HRV signals to compare it to the other methods.

\section{F. DFT of Interpolated Signals (FHPIn, FHRIn, FHTIn)}

The sequence of irregular samples is previously interpolated at regularly spaced time intervals. Then, the FFT is used to calculate the spectrum. We have used spline interpolation with different orders at a sampling frequency of $1 / T \mathrm{~Hz}$. The order of the spline interpolation is shown by the "n" suffix, for example FHRI4 is the abbreviation of this method for cubic interpolation applied to HR signal.

\section{G. AR Method of Interpolated Signals (ARHPIn, ARHRIn, ARHTIn)}

Many authors use parametric AR estimation methods of the PSD in HRV analysis [16]. There are several methods to estimate the $a_{k}$ coefficients of an AR model from the studied time series. Thus, the Yule-Walker, Burg, covariance or modified covariance are different methods for estimating AR coefficients. We used the modified covariance method but we did not observe a significant difference with other AR methods in the HRV analysis. The order used will be indicated in the abbreviation suffix. Thus, ARHRI9 will be a ninth-order AR estimation method applied to the HR interpolated signal. A cubic spline interpolation at a sampling frequency of $1 / T \mathrm{~Hz}$ was used previously to reconstruct an evenly sampled signal from the uneven samples.

\section{Comparison Between the Estimated Spectra}

We carried out two types of experiment in order to compare the different PSD estimates that have been proposed. We made
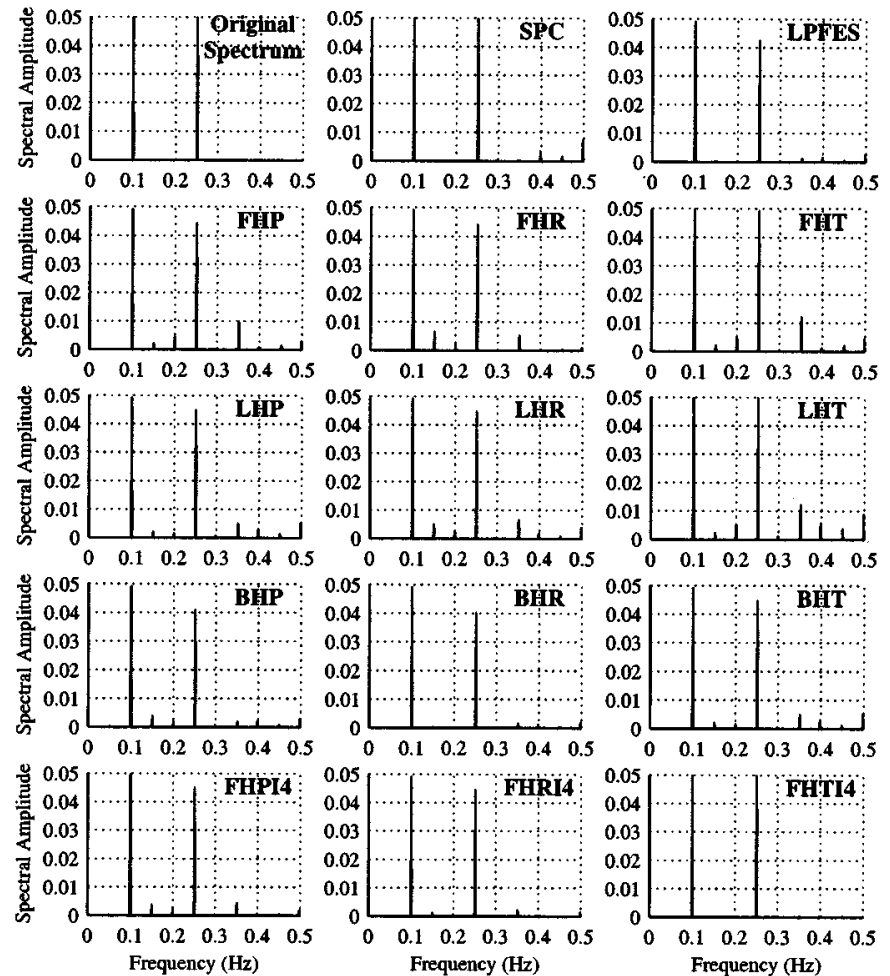

Fig. 4. Amplitude spectra for $m(t)=0.1 \cos (2 \pi \cdot 0.1 \cdot t)+0.1 \cos (2 \pi$. $0.251 \cdot t)$ and $T=1 \mathrm{~s}$ with different PSD estimates. See text for details.

different assumptions with $m(t)$. First, we assumed that $m(t)$ is formed by two-tone function whose frequencies are well known. We intend to corroborate the theoretical results presented in this paper.

The second kind consists of generating the series of beats by means of realistic AR models. We have used for modeling the $m(t)$ signal AR models that approximately match the PSD at supine rest and after head-up tilt described in [8]. Then, the series of beats is generated as output of the IPFM model and the PSD estimation achieved by the described methods.

\section{A. Two-Tone Simulation}

We carried out the same two-tone simulation presented in this paper so as to compare experimentally the results obtained with the previously mentioned theoretical study. In this case, the modulating signal was $m(t)=0.1 \cdot \cos (2 \pi \cdot 0.1 \cdot t)+$ $0.1 \cdot \cos (2 \pi \cdot 0.251 \cdot t)$ with a mean heart period of $T=1 \mathrm{~s}$. We generated 1000 beats following the IPFM model equation. We calculated the different time-domain signals and finally we applied the PSD methods described in this paper. We slightly increased the frequency of the second tone so as to observe the second harmonic spurious contribution $(0.502 \mathrm{~Hz}$ reflected to $0.498 \mathrm{~Hz}$ due to aliasing and with the SPC method coming from $1 / T-2 \cdot 0.251=0.498 \mathrm{~Hz}$ ) and falling into the base band $(0-0.5 \mathrm{~Hz})$. We chose exactly 1000 beats to have an integer number of periods of both tones avoiding spectral leakage due to the finite observation time [28].

Fig. 4 shows the obtained results. In this case, we have presented the amplitude spectra to show both the incorrect amplitude of the tones estimated and the spurious spectral 
TABLE III

COEFFICIENTS AND NOISE VARIANCE OF THE AR MODELS

\begin{tabular}{cccccccccc}
\hline Case & $a_{0}$ & $a_{1}$ & $a_{2}$ & $a_{3}$ & $a_{4}$ & $a_{5}$ & $a_{6}$ & $a_{7}$ & $\sigma^{2}$ \\
\hline Rest & 1 & -1.6265 & 1.8849 & -1.8327 & 1.2970 & -0.7758 & 0.4133 & -0.2136 & $404 \cdot 10^{-6}$ \\
Tilt & 1 & -1.8149 & 2.1365 & -2.1703 & 1.7194 & -0.9221 & 0.5311 & -0.3262 & $137 \cdot 10^{-6}$ \\
\hline
\end{tabular}

components generated. The SPC spectrum has been represented without its unitary delta at $0 \mathrm{~Hz}$. The AR spectral estimates have not been calculated since they are not suitable for tone-like spectral estimation [29].

\section{B. AR Simulation}

To compare the behavior of the spectra estimates to a more realistic signal, we generate the beat series from an $m(t)$ signal following a typical spectrum from a real subject. We have used for modeling the $m(t)$ signal two AR models that approximately match the PSD at supine rest and after head-up tilt described in [8]. The PSD of the AR model is given by

$$
\operatorname{PSD}(f)=\sigma^{2}\left|\frac{1}{A\left(e^{j 2 \pi f}\right)}\right|^{2}
$$

where $\sigma^{2}$ is the variance of the driving white noise with zero mean and $A(z)=\sum_{k=0}^{p} a_{k} \cdot z^{-k}$ is the AR polynomial of order $p$.

Table III shows the coefficients and the variance used for a sampling rate of $1 \mathrm{~Hz}$. The order in both cases was $p=7$. Fig. 5 shows both modeled PSD.

These PSD distributions have been tested, each one with two mean heart periods ( $T=0.8 \mathrm{~s}$ and $T=1.2 \mathrm{~s}$ ). We preferred to keep constant the PSD distributions and change the mean heart period. In relative terms to the mean Nyquist frequency, for the same PSD when $T$ is larger, the high-frequency contribution is larger

In each one of the four cases, we generated 20 random realizations of $N=1024$ samples of the modulating signal, $m[n]$ (sampling frequency of $1 / \mathrm{T} \mathrm{Hz}$ ), following the PSD of each AR model. Then, the $m[n]$ sequences are interpolated obtaining $128 \cdot N$ samples by means of zero padding at its spectra. This operation keeps the spectrum and obtains enough samples to perform the numerical integration involved in the IPFM model. We calculate the cumulative integral of $(1+$ $m[n]) / T$ and we obtain the beat occurrence times, $t_{k}$, as the instants when this integral crosses $k=1,2 \cdots N$. [See Fig. 1(b)]. Additional cubic spline interpolation is carried out in the neighborhood of $t_{k}$ to determine the $t_{k}$ instants with precision. Bearing in mind that the mean of $m[n]$ is zero, we also obtain 1024 beats at $t_{k}$. Finally, the PSD estimation is achieved by all the above described methods. The order of the chosen model of the AR methods for estimation was $p=9$ for the rest case and $p=15$ for the tilt case following the minimum optimal order test [8].

The spectrum of each realization of the $m[n]$ is named "Original spectrum" and is the objective to be estimated. Each realization is considered as an independent recording to estimate its PSD. To show clearly the global behavior of each method
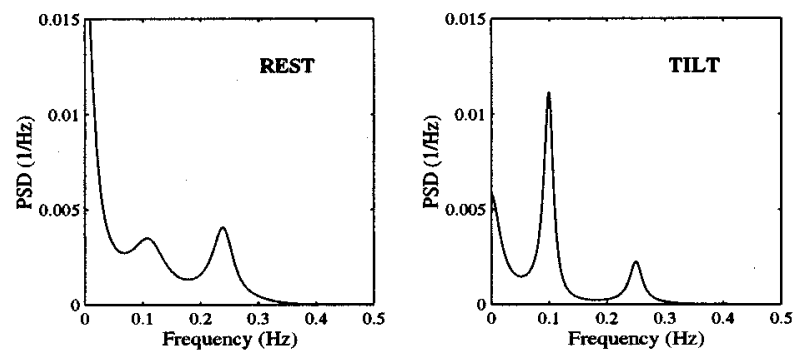

Fig. 5. Modeled PSD of $m(t)$ for the Rest and Tilt cases.

with the frequency, we calculated the mean normalized error $\operatorname{MNE}(f)$ defined as

$$
\operatorname{MNE}(f)=\frac{\sum_{i=1}^{20}\left(\widehat{\operatorname{PSD}}_{i}(f)-\operatorname{PSD}_{i}(f)\right)}{\sum_{i=1}^{20} \operatorname{PSD}_{i}(f)}
$$

where $\operatorname{PSD}_{i}(f)$ is the $\mathrm{PSD}$ of the $i$ th realization of the "Original spectrum" and $\widehat{\mathrm{PSD}}_{i}(f)$ is the PSD estimate with each method on the $i$ th realization. With our sign convention, when the error is positive the power estimation is greater than the original and vice versa. Fig. 6 shows the mean of the 20 realizations of the "Original spectrum" (top left) and the mean of the 20 estimations made with each method (rest of graphics) for the rest case with $T=1.2 \mathrm{~s}$. The modeled PSD is shown as a dashed line and a systematic PSD bias error can be observed in some estimation methods. However, Fig. 7 shows the $\operatorname{MNE}(f)$ of each method and it clearly reflects their behavior with the frequency. We present this case because the PSD estimation methods behave worse when $T$ is larger. The results obtained with the tilt case are quite similar and their graphs are not presented in this paper. The methods based on the HP signal have not been shown since they have a similar or even a worse performance than those based on the HR signal, as can be seen in Fig. 4.

Moreover, to obtain a significant single value of the quality of each method we integrated the absolute value of the error in all the frequencies and we calculated the mean of the normalized error power (MNEP) of each method defined as

$$
\mathrm{MNEP}=\frac{1}{20} \sum_{i=1}^{20} \frac{\int_{-(1 / 2 T)}^{+(1 / 2 T)}\left|\widehat{\operatorname{PSD}}_{i}(f)-\mathrm{PSD}_{i}(f)\right| d f}{\int_{-(1 / 2 T)}^{+(1 / 2 T)} \operatorname{PSD}_{i}(f) d f}
$$

The calculated MNEP is an effective quantitative summary of the estimation methods quality. However, in HRV the PSD is usually divided into different frequency bands [8]. We 

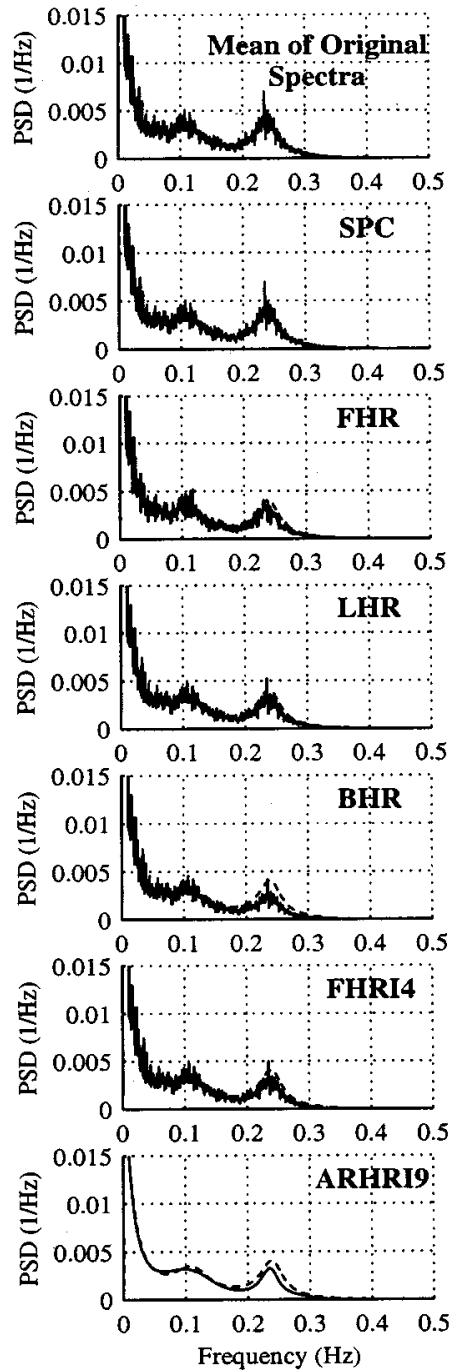
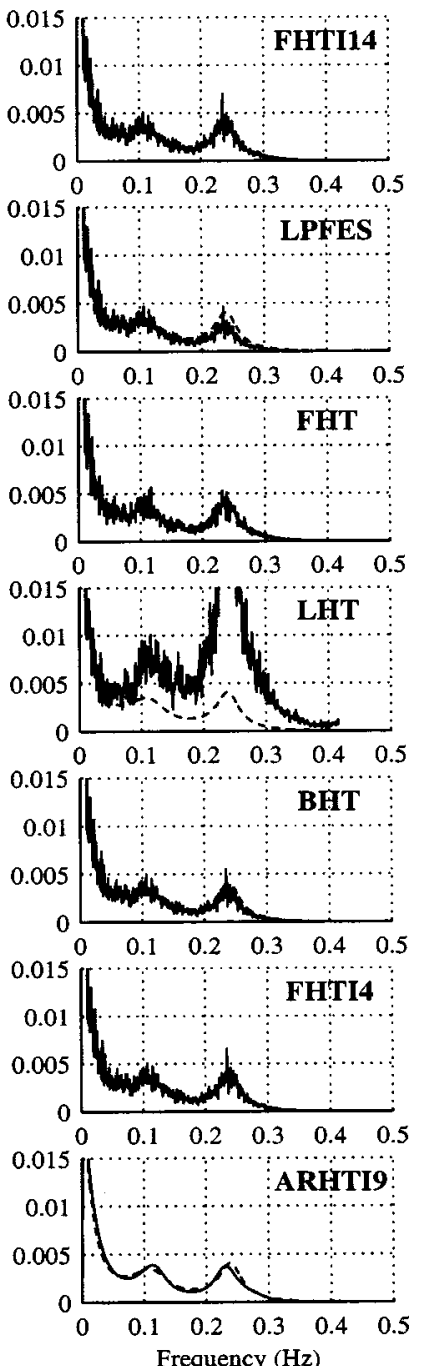

Frequency $(\mathrm{Hz})$

Fig. 6. PSD estimation results for the Rest model and $T=1.2 \mathrm{~s}$. See text for details.

carried out the simulation with 1024 beats that approximately represent between 13 and $20 \mathrm{~min}$ of electrocardiogram (ECG) recording. We used the following bands, recommended in [8], LF $(0.04-0.15 \mathrm{~Hz})$ and HF $(0.15-0.4 \mathrm{~Hz})$. This choice rejects the ULF band $(f<0.003 \mathrm{~Hz})$, with uncertain information, specially with nonstationary recordings, and the UHF band $(f>0.4 \mathrm{~Hz})$ normally involved with noise. The more usual clinical indices are based on the power in each band relative to the sum of the power in the three bands. We calculated the relative power $\mathrm{VLF} / \mathrm{AF}, \mathrm{LF} / \mathrm{AF}$, and $\mathrm{HF} / \mathrm{AF}$ where $\mathrm{AF}$ $=\mathrm{VLF}+\mathrm{LF}+\mathrm{HF}$ and VLF, LF, and $\mathrm{HF}$ is the power in the corresponding band. Then, we calculated the errors $E_{\mathrm{VLF}}=\widehat{\mathrm{VLF}} / \widehat{\mathrm{AF}}-\mathrm{VLF} / \mathrm{AF}, E_{\mathrm{LF}}=\widehat{\mathrm{LF}} / \widehat{\mathrm{AF}}-\mathrm{LF} / \mathrm{AF}$, $E_{\mathrm{HF}}=\widehat{\mathrm{HF}} / \widehat{\mathrm{AF}}-\mathrm{HF} / \mathrm{AF}$ as the difference of the relative power obtained with each method and the one obtained from the original realization of $m[n]$. These errors are signed magnitudes whose sum in the three bands is zero. If the error is positive in one band, the relative power estimated is excessive in this band and vice versa. Thus, the tendency of each method to increase one band power in relation to the others can be easily detected.
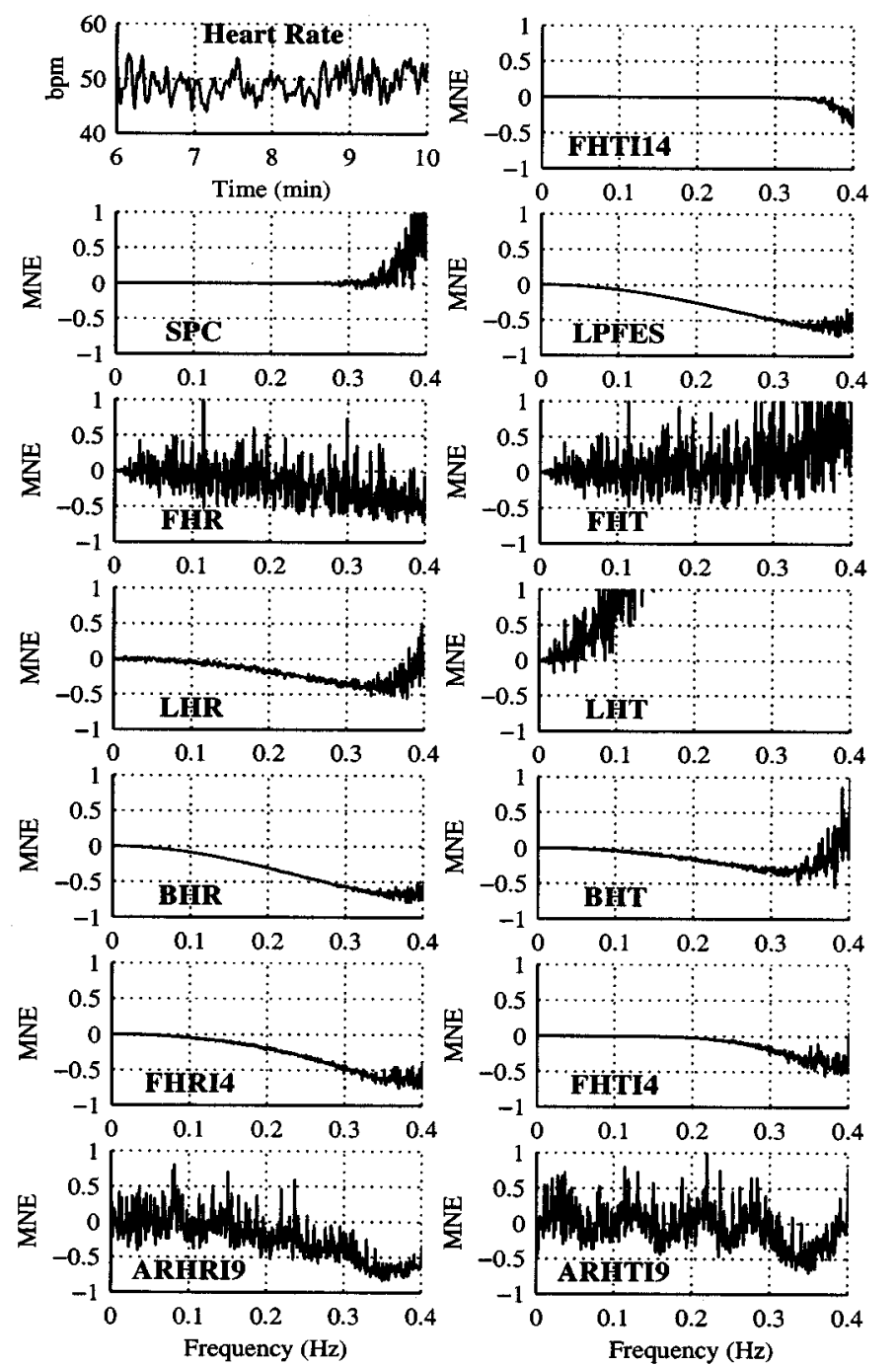

Fig. 7. PSD estimation error for the Rest model and $T=1.2 \mathrm{~s}$. See text for details.

We present in Fig. 8, at the left column the MNEP of the four studies, at the middle column, the mean of $E_{\mathrm{VLF}}, E_{\mathrm{LF}}$, and $E_{\mathrm{HF}}$ and at the right hand column, the standard deviation of these errors. A logarithmic representation of the different magnitudes has been used to represent together the magnitudes in spite of their great differences. In this figure, the methods have been ordered attending to their performance from the left (best) to the right (worse). The methods with a lower performance (FHP, FHR, FHT, and LHT) and those based on the HP signal have not been shown [30].

\section{DISCUSSION}

The above simulations permit us to reinforce the conclusions from Section II-C and to extract some new ones:

As regards the time domain signal used, we observe that both, the HP or the HR signal present a strong and similar low-pass filtering effect due to the fact that the generation of these signals involves this effect inherently (see Figs. 4, 6, and 7). Moreover, these signals do not have a linear dependence with the modulating signal and harmonics appear increasing the noise and dis- 
Case Rest and $T=0.8 \mathrm{~s}$.
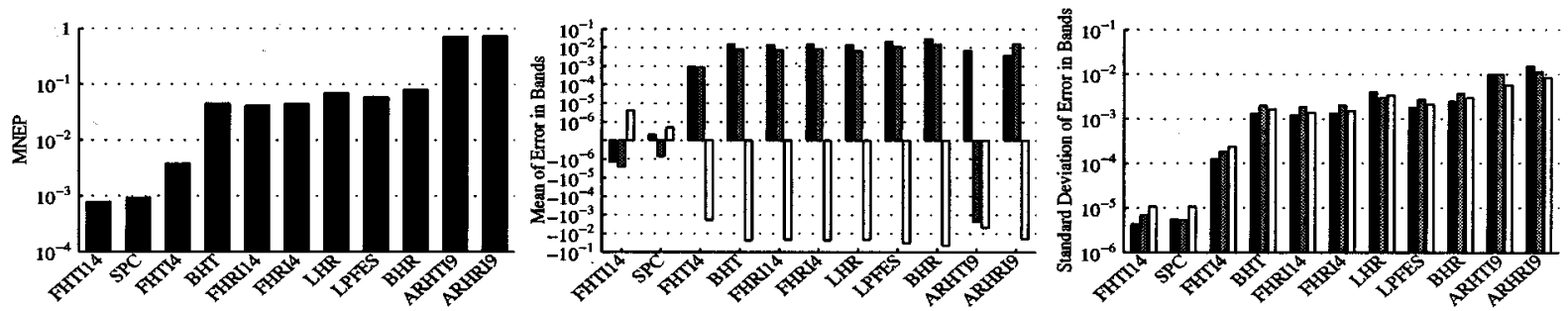

Case Rest and $T=1.2 \mathrm{~s}$.
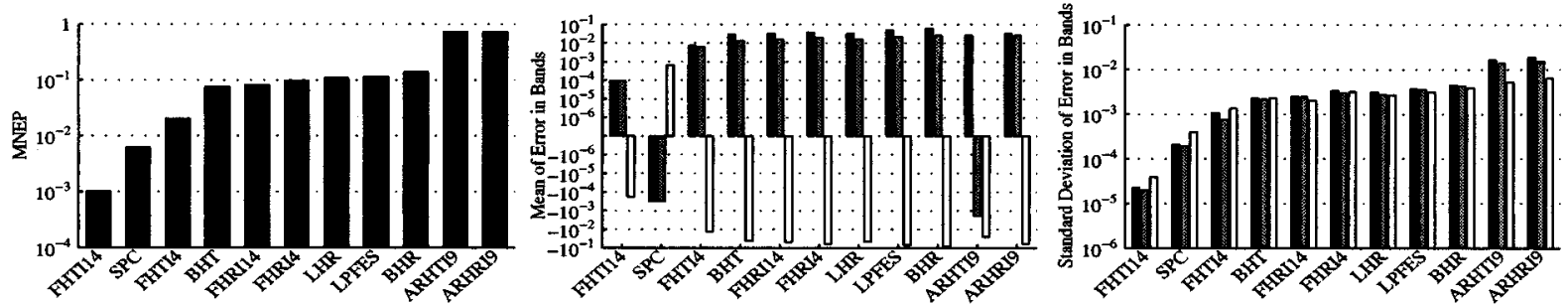

Case Tilt and $T=0.8 \mathrm{~s}$.
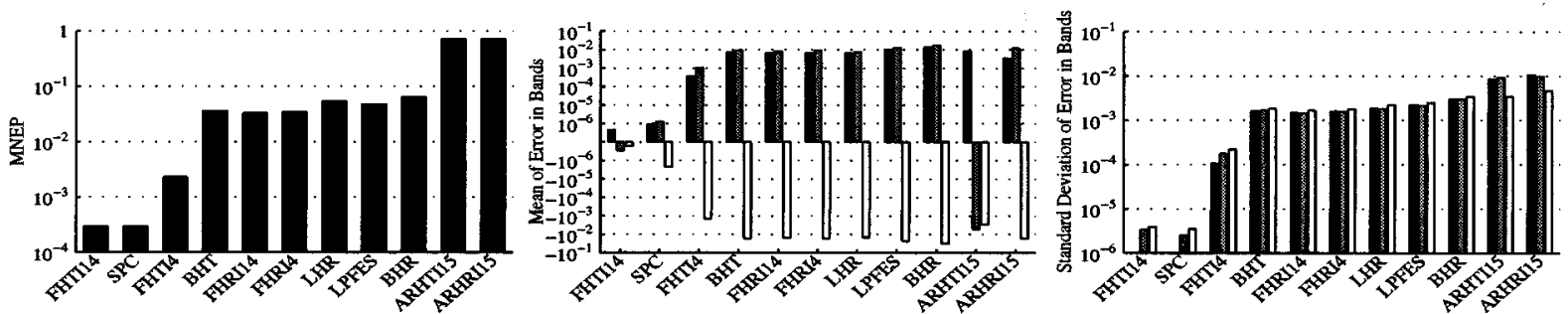

Case Tilt and $T=1.2 \mathrm{~s}$.
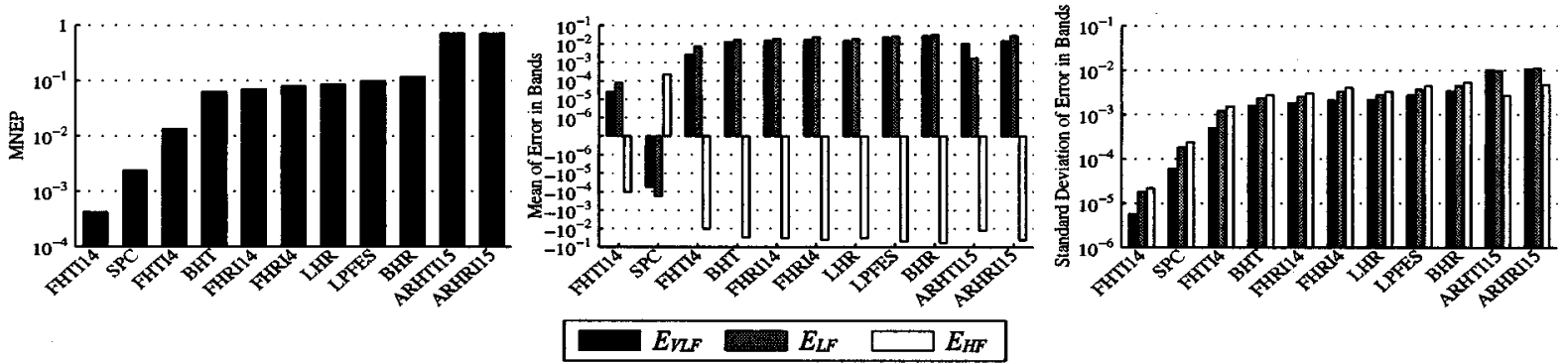

Fig. 8. MNEP, mean, and standard deviation of estimation errors $E_{\mathrm{VLF}}, E_{\mathrm{LF}}$, and $E_{\mathrm{HF}}$ for each method in the four studied cases.

torting the spectra nonlinearly (see Fig. 4). This effect is more noticeable in the HP signal than in the HR signal (see Figs. 1, 2, and 4). However, the HT signal and the Event Series do not present the low-pass filtering effect and the distortion is very low in the Event Series and it is null with the HT signal (see Figs. 4, 6, and 7).

As regards the method used to estimate the PSD, with signals with smooth spectra and relative low power at high frequencies, the SPC method performs well, more effectively than the other classical methods (see Figs. 6 and 7). However, if the spectrum is sharp, spurious components can appear at high frequencies whose amplitude is very sensitive to the original spectral distribution (see Fig. 4). The LPFES method introduces a noticeable low-pass filtering effect due to the insertion of the dummy pulse in the midpoint of each RR interval (see Figs. 6, 7). This can be seen as a process similar to linear interpolation. The direct estimation over the sequences (FHP, FHR, FHT), supposing that are equispaced, introduces a very important nonlinear distortion due to the artificial compression or expansion between beats. They have a poor performance, as it was expected (see Figs. 4, 6, and 7). The direct estimation methods as the Lomb method (LHP, LHR, LHT) also introduce significative distortion due to the convolution with $\operatorname{SPC}(\omega)$ [See (19) and (20)]. Moreover, if the modulating signal has very low-frequency components, as usual with smooth spectra, the LHT method becomes unusable (See Figs. 6 and 7). This is due to the fact that $H T(\omega)=M(\omega) / j \omega$ has very high amplitudes at low frequencies. When $\operatorname{LHT}(\omega)$ is multiplied by $j \omega$ to recover $M(\omega)$, the first term in (20) will be the correct $M(\omega)$ but the second term will be not negligible. It will have the $H T(\omega)$ high amplitudes displaced due to the convolution with $M(\omega)$. The sum of both terms increases the amplitude of the high-frequency components strongly that afterwards need even be multiplied by $j \omega$. This can be summarized as

$$
j \omega \cdot \operatorname{LHT}(\omega)=M(\omega)+j \omega \cdot\left(\frac{M(\omega)}{j \omega} * M(\omega)\right) \nRightarrow M(\omega)
$$


The Berger method (BHP, BHR, BHT) reduces high-frequency contamination of the HP, HR, or HT but it presents an added low-pass filtering effect in all cases (see Figs. 4, 6, and 7). This is because, even this method corrects the filtering effect of the convolution with the rectangular window, it cannot correct the time-variant low-pass filtering due to the "sample and hold" process involved. Methods based on spline interpolation present a reduced low-pass filtering effect due to the interpolation, which has been practically eliminated by increasing the order of the spline (see Figs. 6 and 7). The results obtained with the AR methods present inferior performance than those nonparametric ones in spite of the simulated signal was generated through an AR model. The obtained mean error of the clinical indices and the standard deviation was larger than with the other methods (see Fig. 8). These results are in agreement with those obtained in [31].

We demonstrated that a robust interpolation method in conjunction with the HT signal presents the best HRV estimation. The improvement relative to the SPC is more noticeable when the power at high frequencies is greater, or for the same PSD distribution, when the mean heart period is larger. This condition in the cases studied is met when $T=1.2 \mathrm{~s}$. and effectively, in these cases the performance of the SPC method clearly worsens (see Fig. 8). In general, when the original PSD has higher relative power at high frequencies, more error will be introduced.

All these effects are reflected in the estimate of the clinical indices as it is summarized in Fig. 8.

The previous cases were simulated with great precision in the beats position $\left(t_{k}\right)$. In real situations, the determination of the fiducial point of the QRS complex position involves errors due to noise or low sampling frequency of the ECG recording. We made the same simulation but reducing the resolution of the beat occurrence times. This is equivalent to add white noise uniformly distributed over the original $t_{k}$ positions. The white noise introduced in a time-domain signal becomes colored in the PSD spectral estimation, increasing quadratically with the frequency [32]. In [32], it is shown for the HP signal that a decreased sampling frequency in ECG increases the noise power at high frequencies in PSD estimation. This occurs in all estimation methods presented in this paper [30]. The best performance methods (FHTI and SPC) were more sensitive to the low resolution simply because these methods have no biased error, and the methods with poorer performance did not suffer this error appreciably because they have a significant biased estimation error. Thus, to keep the performance of the best methods it is needed to detect the position of each QRS complex with a precision better than $0.25 \mathrm{~ms}$. This refinement can be accomplished by interpolating the ECG on the neighborhood of each QRS.

\section{CONCLUSION}

In this paper, we present a study analyzing the problems of the application of the different time domain signals used in HRV assuming the IPFM model. We introduced the new HT signal that overcomes these problems. We analyzed the irregular sampling problem and we show that an adequate interpolation method with appropriate frequency response is a better solution than direct estimation methods as Lomb method. We carried out sim- ulations based on AR models with PSD considered as standard in real subjects. In these simulations we compared most of the methods known to us. Results show how the method based on the interpolation of the HT signal has achieved the best results followed by the SPC method. It is needed to detect the position of each QRS complex with a precision better than $0.25 \mathrm{~ms}$. to maintain the performance of the best methods.

In summary, we may conclude that to study HRV assuming the IPFM model, the time-domain signal that better recovers the modulation properties of the sino-atrial node is the HT signal. The PSD estimation method that gives the best modulation spectrum estimates is the Fourier transform of the HT signal interpolated by high order splines. This technique get the lower error in the estimate of the clinical indices measured as PSD at the VLF, LF, and HF bands as used in clinical studies. Moreover, the practical null distortion achieved by the high order spline interpolation of the HT signal make it suitable for a continuous time-domain estimate of the instantaneous heart rate. This technique will allow to use time frequency methods in situations dealing with nonstationary ECG recording, such as stress test.

\section{APPENDIX \\ SPC AT THE BASE BAND}

The $\operatorname{spc}(t)$ signal represents a problem equivalent to the problem of pulse position modulation (PPM) which appears in modulation systems [21]. In [21], it is shown that

$$
\begin{aligned}
& \sum_{k=-\infty}^{\infty} \delta\left(t-k T+\Delta\left(t_{k}\right)\right) \\
& \quad=\frac{\left|1+\Delta^{\prime}(t)\right|}{T}\left\{1+2 \sum_{n_{0}=1}^{\infty} \cos \left(\frac{2 \pi n_{0}}{T}(t+\Delta(t))\right)\right\}
\end{aligned}
$$

We can rewrite (13) using (3) as

$$
\operatorname{spc}(t)=\sum_{k=-\infty}^{\infty} \delta\left(t-k T+h t\left(t_{k}\right)\right) .
$$

In our case, $\Delta(t)=h t(t), \Delta^{\prime}(t)=m(t)$ and $|m(t)|<1$. Thus, using (25)

$$
\begin{aligned}
\operatorname{spc}(t)= & \frac{1+m(t)}{T} \\
& \cdot\left\{1+2 \sum_{n_{0}=1}^{\infty} \cos \left(\frac{2 \pi n_{0}}{T}(t+h t(t))\right)\right\}
\end{aligned}
$$

and the spectrum of $\operatorname{spc}(t)$ is

$$
\begin{aligned}
\operatorname{SPC}(\omega)=\frac{1}{T} & (\delta(\omega)+M(\omega)) \\
& *\left\{\delta(\omega)+2 \sum_{n_{0}=1}^{\infty}\right. \\
& \left.\cdot \mathcal{F} \mathcal{T}\left\{\cos \left(\frac{2 \pi n_{0}}{T}(t+h t(t))\right)\right\}\right\} .
\end{aligned}
$$

The more important spectral contamination at the base band is given by the fundamental frequency modulation, that is, for 
$n_{0}=1$. Greater values of $n_{0}$ require greater convolution orders to reach the base band and its amplitude will be very small. Then, at the base band the spectrum can be approximated as

$$
\operatorname{SPC}(\omega) \approx \frac{1}{T}\{\delta(\omega)+M(\omega)+\operatorname{FM}(\omega)+M(\omega) * \operatorname{FM}(\omega)\}
$$

where $\operatorname{FM}(\omega)$ is given by

$$
\operatorname{FM}(\omega)=\mathcal{F} \mathcal{T}\left\{2 \cdot \cos \left(\frac{2 \pi}{T} t+\frac{2 \pi}{T} h t(t)\right)\right\}
$$

\section{REFERENCES}

[1] B. Sayers, "Analysis of heart rate variability," Ergonomics, vol. 16, pp. $17-32,1973$

[2] S. Akselrod and D. Gordon et al., "Power spectrum analysis of heart rate fluctuations: A quantitative probe of beat-to-beat cardiovascular control," Science, vol. 213, pp. 220-222, 1981.

[3] B. Pomerantz and R. J. Macaulay et al., "Assessment of autonomic function in humans by heart rate espectral analysis," Amer. J. Physiol., vol. 248, pp. H151-H153, 1985.

[4] M. Pagani and F. Lombardi et al., "Power spectral analysis of heart rate and arterial pressure variabilities as a marker of sypatho-vagal interaction in man and conscious dog," Circ. Res., vol. 59, pp. 178-193, 1986.

[5] R. E. Klieger, J. P. Miller, J. T. Bigger, and A. J. Moss, "Decreased heart rate variability and its association with increased mortality after myocardial infarction," Amer. J. Cardiol., vol. 59, pp. 256-262, 1987.

[6] T. van den Akker, A. S. M. Koeleman, L. A. H. Hogenhuis, and O. Rompelman, "Heart rate variability and blood pressure oscillations in diabetics with autonomic neuropathy," Automedica, pp. 201-208, 1983.

[7] G. Myers, G. Martin, N. Magid, P. Barnett, J. Schaad, J. Weiss, M. Lesch, and D. H. Singer, "Power spectral analysis of heart rate variability in sudden cardiac death: Comparison to other methods," IEEE Trans. Biomed. Eng., vol. BME-33, pp. 1149-1156, Dec. 1986.

[8] T. F. of The ESC/ASPE, "Heart rate variability: Standards of measurement, phisiological interpretation, and clinical use," Ann. Noninvasive Electrocardiol., vol. 1, pp. 151-181, Apr. 1996.

[9] E. J. Bayly, "Spectral analysis of pulse frequency modulation in the nervous systems," IEEE Trans. Biomed. Eng., vol. BME-15, pp. 257-265, Oct. 1968.

[10] O. Rompelman, J. B. Snijders, and C. van Spronsen, "The measurement of heart rate variability spectra with the help of a personal computer," IEEE Trans. Biomed. Eng., vol. BME-29, pp. 503-510, July 1982.

[11] O. Rompelman, A. Coenen, and R. Kitney, "Measurement of heart-rate variability: Part 1 -Comparative study of heart-rate variability analysis methods," Med. Biol. Eng. Comput., vol. 15, pp. 239-252, 1977.

[12] R. W. DeBoer, J. M. Karemaker, and J. Strackee, "Spectrum of a series of point event, generated by the integral pulse frequency modulation model," Med. Biol. Eng. Comput., vol. 23, pp. 138-142, 1985.

[13] —, "Comparing spectra of a series of point events particularly for heart rate variability data," IEEE Trans. Biomed. Eng., vol. BME-31, pp. 384-387, Apr. 1984.

[14] R. D. Berger, S. Akeselrod, D. Gordon, and R. J. Cohen, "An efficient algorithm for spectral analysis of heart rate variability," IEEE Trans. Biomed. Eng., vol. BME-33, pp. 900-904, Sept. 1986.

[15] P. Castiglioni, "Evaluation of heart rhythm variability by heart or heart period: Differences, pitfalls and help from logarithms," Med. Biol. Eng. Comput., vol. 33, pp. 323-330, 1995.

[16] L. T. Mainardi, A. M. Bianchi, G. Baselli, and S. Cerutti, "Pole-traking algorithms for the extraction of time variant heart rate variability spectral parameters," IEEE Trans. Biomed. Eng., vol. 42, pp. 250-259, Mar. 1995.

[17] J. Mateo and P. Laguna, "New heart rate variability time-domain signal construction from the beat occurrence time and the IPFM model," in Computers in Cardiology. Los Alamitos, CA: IEEE Computer Soc. Press, 1996, pp. 185-188.

[18] — - "Improved interpolation of unevenly sampled heart rate signals," in Computers in Cardiology. Los Alamitos, CA: IEEE Computer Soc. Press, 1997, pp. 137-140.

[19] B. P. Demidovich and I. A. Maron, Computational Mathematics. Moscow, Russia: MIR, 1981
[20] P. Laguna, G. B. Moody, and R. G. Mark, "Power spectral density of unevenly sampled data by least-square analysis: Performance and application to heart rate signals," IEEE Trans. Biomed. Eng., vol. 45, pp. 698-715, June 1998

[21] A. B. Carlson, Communication Systems. An Introduction to Signal and Noise in Electrical Communication, 3rd ed. New York: MacGraw Hill, 1986.

[22] M. Nakao, M. Norimatsu, Y. Mizutani, and M. Yamamoto, "Spectral distorsion properties of the integral pulse frequency modulation model," IEEE Trans. Biomed. Eng., vol. 44, pp. 419-426, May 1997.

[23] C. de Boor, A Practical Guide to Splines. New York: Springer-Verlag, 1978.

[24] N. R. Lomb, "Least-squares frequency analysis of unequaly spaced data," Astrophysical Space Sci., vol. 39, pp. 447-462, 1976.

[25] J. D. Scargle, "Studies in astronomical time series analysis II. statistical aspects of spectral analisis of unevenly spaced data," Astrophisical J., vol. 263, pp. 835-853, 1982

[26] W. H. Press and G. B. Rybicki, "Fast algorithm for spectral analysis of unevenly sampled data," Astrophysical J., vol. 338, pp. 277-280, 1989.

[27] W. H. Press, S. A. Teukolsky, W. T. Vetterling, and B. P. Flannery, Numerical Recipes in C: The Art of Scientific Computing, 2nd ed. New York: Cambridge Univ. Press, 1992.

[28] A. V. Oppenheim and R. W. Schafer, Digital Signal Processing. Englewood Cliffs, NJ: Prentice-Hall, 1975.

[29] J. Proakis and D. G. Manolakis, Introduction to Digital Signal Processing. New York: Macmillan, 1988.

[30] J. Mateo, "Análisis de la variabilidad del ritmo cardiaco: Representación temporal e índices clínicos," Ph.D. thesis (in Spanish), Universidad de Zaragoza, Zaragoza, Spain, 2000.

[31] D. J. Christini, A. Kulkarni, S. Rao, E. Stuttman, F. M. Bennet, K. Lutchen, J. M. Hausdorff, and N. Oriol, "Uncertainty of AR spectral estimates," in Computers in Cardiology. Los Alamitos, CA: IEEE Computer Soc. Press, 1993, pp. 451-454.

[32] M. Merri, D. Farden, J. M. Titlebaum, and E. L. Titlebaum, "Sampling frequency of the electrocardiogram for spectral analysis of the heart rate variability,” IEEE Trans. Biomed. Eng., vol. 37, pp. 99-105, Dec. 1990.

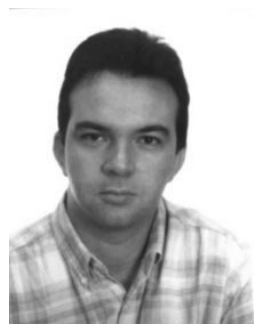

Javier Mateo was born in Zaragoza, Spain, in 1964. He received the M.Sc. degree in electrical engineering in 1989 from the Polytechnic University of Madrid (U.P.M.), Madrid, Spain, and the Ph.D. degree in 2000 from the University of Zaragoza (U.Z.), Zaragoza, Spain.

From 1989 to 1993, he was with Cables de Comunicaciones S. A. where he worked on fiber optic sensors and optical communications. In 1993, he joined Electronic Engineering and Communications department of the University of Zaragoza, where he developed his Ph.D. thesis. He is currently Assistant Professor of Optical Fiber Communications in the Department of Electronic Engineering and Communications at the Polytechnic Center of the University of Zaragoza. His professional research interests are in signal processing, in particular applied to biomedical signals, fiber optic sensors, and optical communication systems.

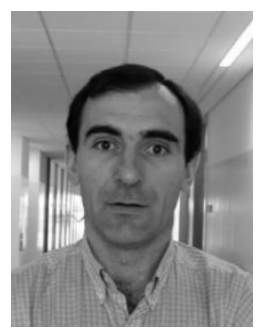

Pablo Laguna was born in Jaca (Huesca), Spain, in 1962. He received the MS degree in physics and the Ph.D. degree from the University of Zaragoza (U.Z.), Spain, in 1985 and 1990, respectively. The Ph.D. thesis was developed at the Biomedical Engineering Division of the Institute of Cybernetics (I.C.), Polytechnic University of Catalonia (U.P.C.)-C.S.I.C., Barcelona, Spain.

He is currently an Associated Professor of Signal Processing and Communications in the Department of Electronic Engineering and Communications at the Centro Politécnico Superior, U.Z. From 1987 to 1992, he worked as Assistant Professor in the Department of Control Engineering at the U.P.C., and as a Researcher at the Biomedical Engineering Division of the I.C. His professional research interests are in signal processing, in particular applied to biomedical applications. 